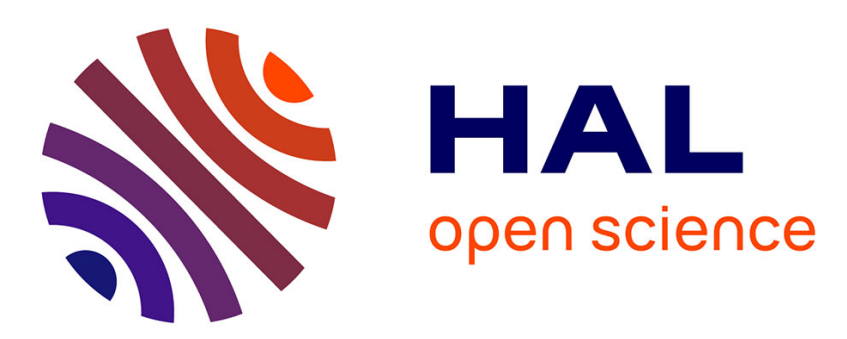

\title{
Tests of the Standard Model and $\alpha\left(m^{2} \_Z\right)$
}

B. Pietrzyk

\section{To cite this version:}

B. Pietrzyk. Tests of the Standard Model and $\alpha\left(m^{2} \_Z\right)$. II International Workshop on e+e- Collisions from phi to psi, Feb 2006, Novosibirsk, Russia. pp.18-21, 10.1016/j.nuclphysbps.2006.09.059 _ in2p300117377

\section{HAL Id: in2p3-00117377 https://hal.in2p3.fr/in2p3-00117377}

Submitted on 1 Dec 2006

HAL is a multi-disciplinary open access archive for the deposit and dissemination of scientific research documents, whether they are published or not. The documents may come from teaching and research institutions in France or abroad, or from public or private research centers.
L'archive ouverte pluridisciplinaire HAL, est destinée au dépôt et à la diffusion de documents scientifiques de niveau recherche, publiés ou non, émanant des établissements d'enseignement et de recherche français ou étrangers, des laboratoires publics ou privés. 


\section{Tests of the Standard Model and $\alpha\left(\mathrm{m}_{\mathrm{Z}}^{2}\right)$}

\section{B. Pietrzyk ${ }^{\mathrm{a} *}$}

a Laboratoire de Physique des Particules (LAPP), IN2P3-CNRS,

F-74019 Annecy-le-Vieux, France

Tests of the Standard Model and influence of $\alpha\left(\mathrm{m}_{\mathrm{Z}}^{2}\right)$ on these tests are discussed.

The final results on precision measurements at the $\mathrm{Z}$ pole are recently published 10 years after the end of measurements at LEP1. The LEP/SLD measurements had to take into account huge ISR corrections which were calculated with the precision $\sim 10^{-4}$. The precision of measurements to $\sim 10^{-3}$ exceeded sufficiently the natural size of genuine ElectroWeak (EW) corrections

$\frac{\alpha\left(\mathrm{m}_{\mathrm{Z}}^{2}\right)}{\pi \sin ^{2} \theta} \sim 1 \%$

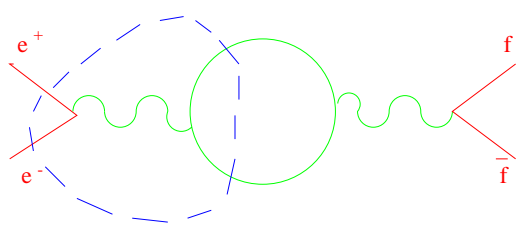

Figure 1. The effective coupling.

The notion of effective couplings has been introduced in which radiative corrections, both to the vertex and to the propagator, have been included. The precise EW measurements included the $\mathrm{Z}$ line-shape measurement, the asymmetries, $\tau$ polarisation from LEP1, the left-right asymmetries from SLD, $\mathrm{m}_{\mathrm{W}}$ and $\Gamma_{\mathrm{W}}$ from LEP2 and Tevatron and $\mathrm{m}_{\mathrm{t}}$ from Tevatron. The asymmetries and $\tau$ polarisation results have been converted into the $\sin ^{2} \Theta_{\text {eff }}^{\text {lept }}$ measurements. An interesting feature of $\sin ^{2} \Theta_{\text {eff }}^{\text {lept }}$ measurements is that there is a $3 \sigma$ difference between measurements made with leptons and quarks.

$\overline{\text { *pietrzyk@lapp.in2p3.fr }}$
The radiative corrections have been divided in two parts: the running of $\alpha(\mathrm{QED})$ and genuine EW radiative corrections. An example, made in 1996, is shown in Fig. 2 [1].

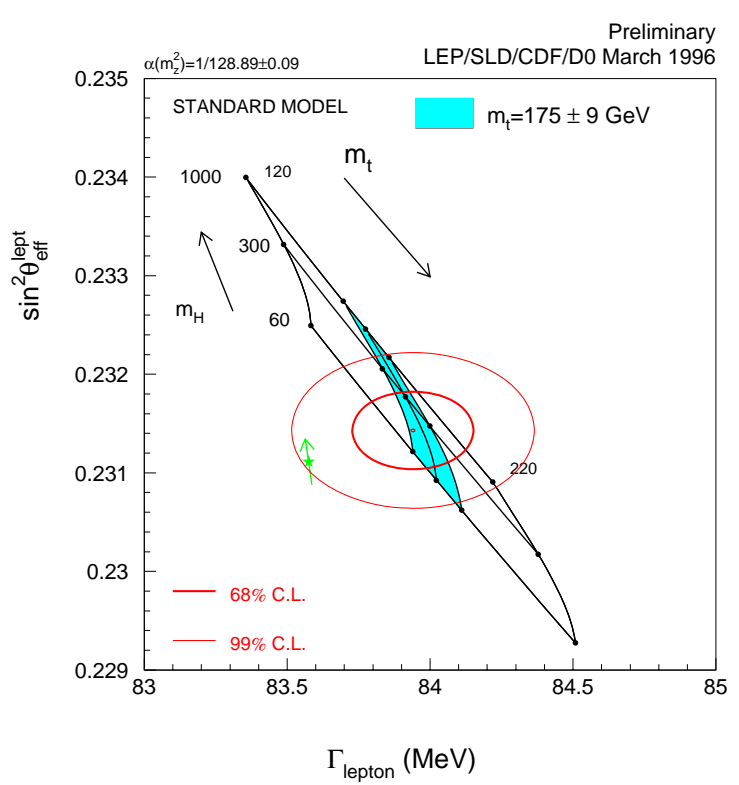

Figure 2. $\sin ^{2} \Theta_{\text {eff }}^{\text {lept }}$ versus leptonic $Z$ width $\Gamma_{\text {lepton. }}$

The star shows the prediction of the SM if among radiative corrections only running of $\alpha$ is included. The value of alpha at the $\mathrm{Z}$ pole, $\alpha\left(\mathrm{m}_{\mathrm{Z}}^{2}\right)$, is not any more $1 / 137 .(), \alpha(0)$, but $1 / 128.940 \pm 0.048, \alpha(s),[2]$.

$$
\alpha(\mathrm{s})=\frac{\alpha(0)}{1-\Delta \alpha_{\mathrm{l}}(\mathrm{s})-\Delta \alpha_{\text {had }}^{(5)}(\mathrm{s})-\Delta \alpha_{\mathrm{t}}(\mathrm{s})}
$$


The contribution of leptonic loops to the running of alpha, $\Delta \alpha_{1}(\mathrm{~s})$, is calculated with a practically negligible uncertainty. The top quark contribution, $\Delta \alpha_{\mathrm{t}}(\mathrm{s})$, is calculated separately since the value of the top quark mass is a result of the SM fit. The contribution of the other 5 quark loops, "hadronic contribution to the running of $\alpha ", \Delta \alpha_{\text {had }}^{(5)}\left(\mathrm{m}_{\mathrm{Z}}^{2}\right)=0.02758 \pm 0.00035[2]$ at the $\mathrm{Z}$ pole, is calculated by integrating the experimentally measured $\mathrm{R}_{\text {had }}=\frac{\sigma_{\text {had }}}{\sigma_{\mu \mu}}$ in $\mathrm{e}^{+} \mathrm{e}^{-}$annihilation (Fig. 3).

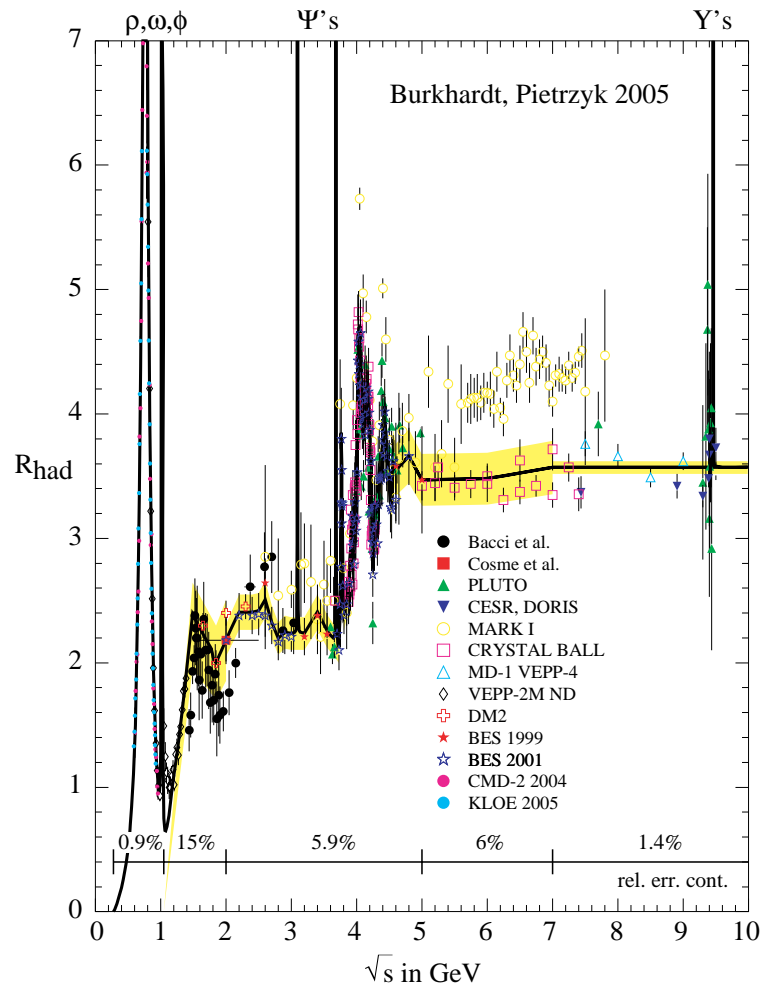

Figure 3. $\mathrm{R}_{\text {had }}$.

The genuine EW radiative corrections depend strongly $\left(\sim \mathrm{m}_{\mathrm{t}}^{2}\right)$ on the top mass and weaker $\left(\sim \ln \frac{\mathrm{m}_{\mathrm{H}}^{2}}{\mathrm{~m}_{\mathrm{W}}^{2}}\right)$ on the Higgs mass, as shown in Fig. 2. Therefore the prediction of the value of the top mass was the main result of the SM fits, before its value was measured at Tevatron in 1994, as shown in Fig. 4. In fact, the prediction of the SM fits for the top mass, just before it was measured, was $177_{-11-19}^{+11+18} \mathrm{GeV}[3]$.

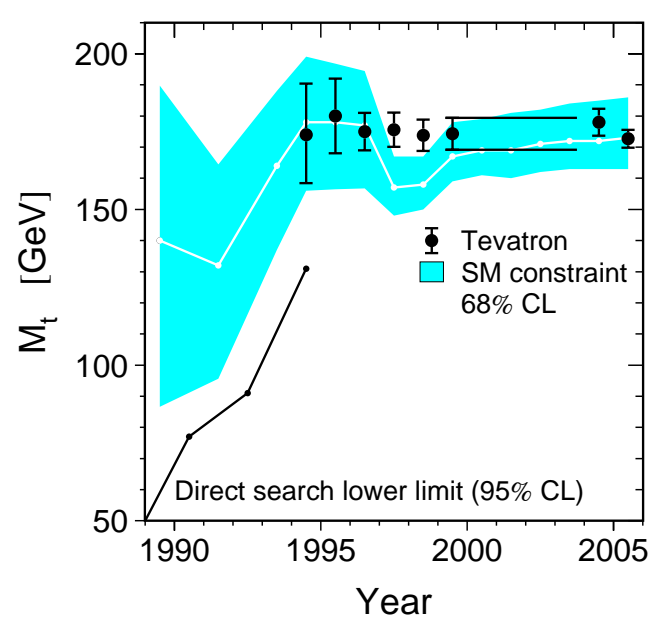

Figure 4. Direct and indirect determination of the top quark mass as a function of time.

The region of the SM predictions is strongly reduced when the top mass value is known as indicated by the shadowed region on Fig. 2. The prediction of the value of the Higgs mass becomes then the main result of the SM fits. The winter 2006 fit result is shown on Fig. 5.

The preferred value of the Higgs mass, corresponding to the minimum of the "blue band curve", is $89_{-30}^{+42}[1]$, the one-sided $95 \%$ confidence level upper limit is $175 \mathrm{GeV}$. The resent variation of the fitted value of the Higgs mass are caused by the progress in the top mass measurements as seen in Table 1.

\section{Table 1}

The recent variation of the fitted value of the Higgs mass with the value of the measured top mass

\begin{tabular}{rrrr}
\hline year & $\begin{array}{r}\mathrm{m}_{\mathrm{t}} \\
(\mathrm{GeV})\end{array}$ & $\begin{array}{r}\mathrm{m}_{\mathrm{H}} \\
(\mathrm{GeV})\end{array}$ & $\begin{array}{r}\text { upper limit } \\
(\mathrm{GeV})\end{array}$ \\
\hline 2003 & $174.3 \pm 5.1$ & $96_{-38}^{+60}$ & 219 \\
2004 & $178.0 \pm 4.3$ & $114_{-45}^{+69}$ & 260 \\
2005 & $172.7 \pm 2.9$ & $91_{-32}^{+45}$ & 186 \\
2006 & $172.5 \pm 2.3$ & $89_{-30}^{+42}$ & 175 \\
\hline
\end{tabular}

The role of $\alpha\left(\mathrm{m}_{\mathrm{Z}}^{2}\right)$ in the SM fits can be understood from Fig. 2. If the value of $\alpha\left(\mathrm{m}_{\mathrm{Z}}^{2}\right)$ (star) is changed, the SM prediction is shifted with respect to the experimental results causing the change in 


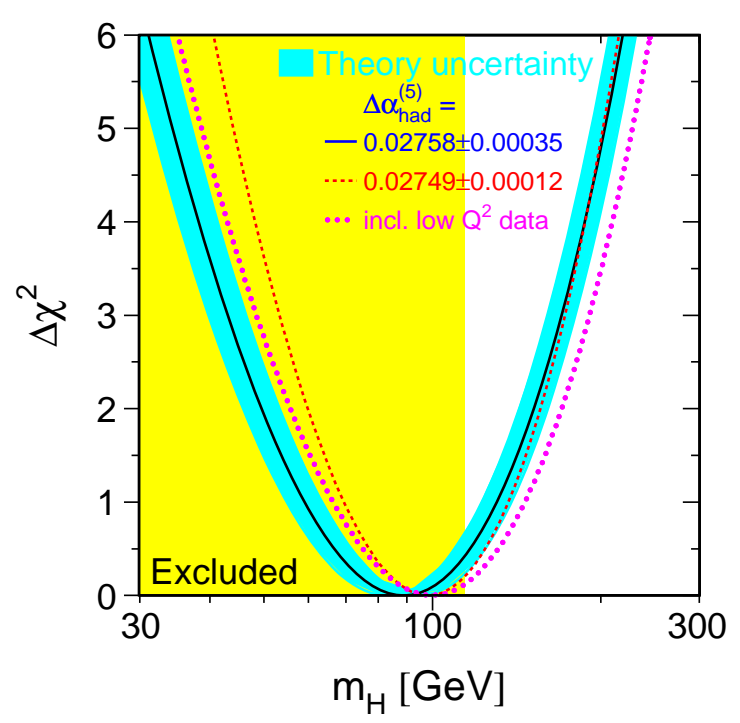

Figure 5. $\Delta \chi^{2}$ as a function of Higgs mass $\mathrm{m}_{\mathrm{H}}$.

the prediction for the Higgs mass.

The prediction of the SM fits in the plane $\alpha\left(m_{\mathrm{Z}}^{2}\right)-\mathrm{m}_{\mathrm{H}}$, when $\alpha\left(\mathrm{m}_{\mathrm{Z}}^{2}\right)$ is removed from the fit input, is shown on Fig. 6. A strong correlation is observed. It is interesting to observe that the SM prediction for the hadronic contribution to the running of $\alpha$ coincides well with the value obtained from the integration of $\mathrm{R}_{\text {had }}$ in the region of $\mathrm{m}_{\mathrm{H}}$ still compatible with the direct searches.

There were mainly two major changes in the history of determination of $\Delta \alpha_{\text {had }}^{(5)}$, as shown on Fig. 7. The Crystal Ball measurements [4] in $\mathrm{e}^{+} \mathrm{e}^{-}$c.m.s. energy region between 5 and 7.4 $\mathrm{GeV}$, used in 1995 evaluation of $\Delta \alpha_{\text {had }}^{(5)}$, significantly lowered the $\mathrm{R}$ values reported previously by the MARK I Collaboration [5], as seen on Fig. 3 . The BES measurements in the energy region between 2 and $5 \mathrm{GeV}$, first reported in ICHEP 2000 Conference in Osaka [6,7], caused the shift of the Higgs mass prediction by about $30 \mathrm{GeV}[8]$.

The recent measurements of the CMD2 [9] and KLOE [10] Collaboration in the $\rho$ region shown in Fig. 8 had only a very minor impact since the previous measurement were already relatively precise in this $\mathrm{e}^{+} \mathrm{e}^{-}$c.m.s. energy region. In our analysis [2] we have integrated separately CMD2 and KLOE points and then combined the results of

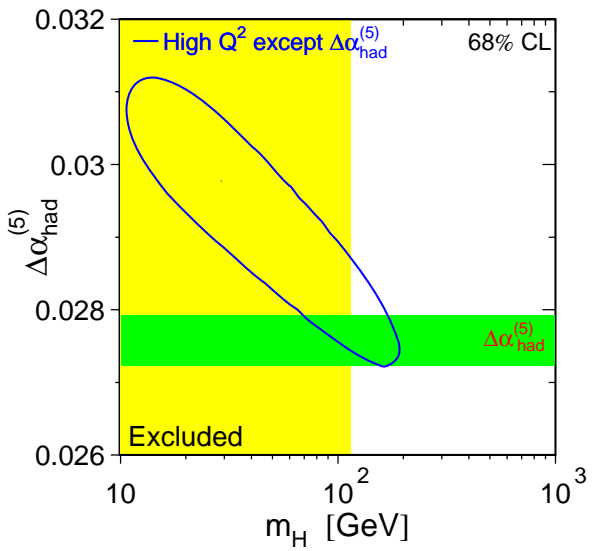

Figure 6. Contour curve of $95 \%$ probability in the $\left(\Delta \alpha_{\text {had }}^{(5)}\left(\mathrm{m}_{\mathrm{Z}}^{2}\right), \mathrm{m}_{\mathrm{H}}\right)$ plane, when $\Delta \alpha_{\text {had }}^{(5)}\left(\mathrm{m}_{\mathrm{Z}}^{2}\right)$ is removed from the fit input.

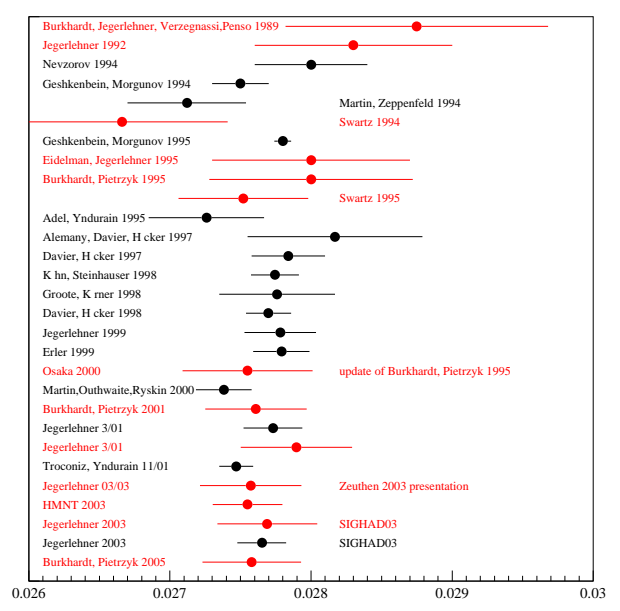

Figure 7. History of evaluation of $\Delta \alpha_{\text {had }}^{(5)}$.

integration. Similarly adding recently published SND [11] results changes $\Delta \alpha_{\text {had }}^{(5)}$ by less than $\frac{1}{10} \sigma$. The revised SND results [12] would change the $\Delta \alpha_{\text {had }}^{(5)}$ even less.

Fig. 9 shows the relative contribution of different $\mathrm{e}^{+} \mathrm{e}^{-}$c.m.s. energy regions to $\Delta \alpha_{\text {had }}^{(5)}$ both in magnitude and uncertainty. The region between 1.05-2 GeV has important contribution to the uncertainty despite its small contribution to the magnitude. Improving the precision of measurements from $15 \%$ (Fig. 3) to $5 \%$ would change the total uncertainty on $\Delta \alpha_{\text {had }}^{(5)}$ from 0.00035 to 
4

hadvac 2005 15.2. $618 \mathrm{H} 53$

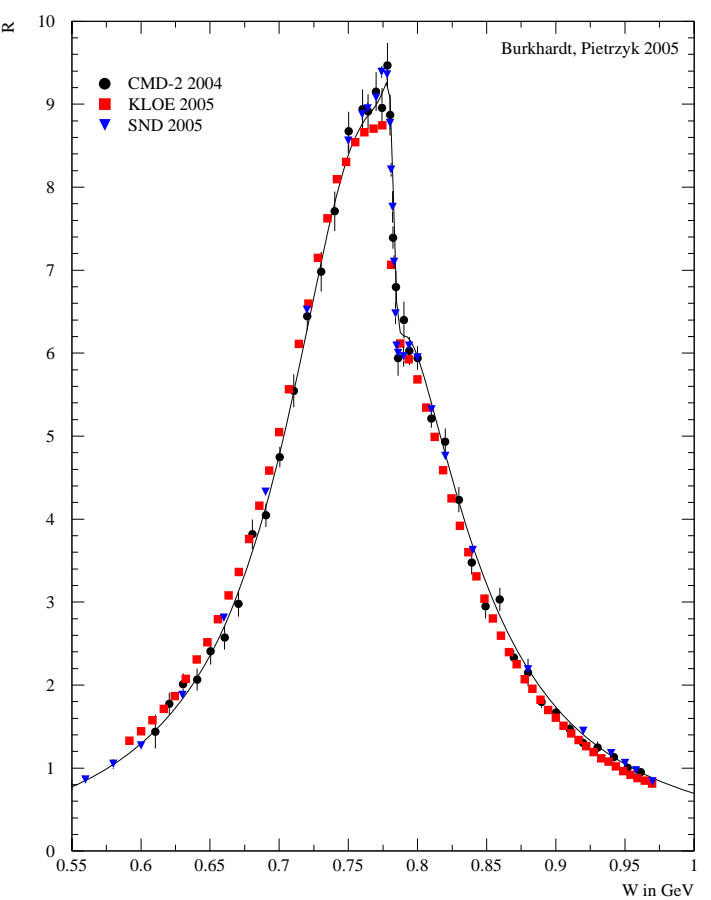

Figure 8. CMD2, KLOE and SND measurements.

0.00027 . The change in the fitted value of the Higgs mass would be small. However, the change $\mathrm{R}_{\text {had }}$ by $\pm 1 \sigma$ in this c.m.s. energy region would shift the central value of the fitted Higgs mass by ${ }_{-9}^{+16} \mathrm{GeV}$. Therefore more precise measurements in this $\mathrm{e}^{+} \mathrm{e}^{-}$c.m.s. energy region are important.

LHC will start at 2007. Will the prediction from the SM for $m_{H}$ be as successful as it was for $\mathrm{m}_{\mathrm{t}}$ ?

I would like to thank the organizers for inviting me to come to this very interesting and important workshop and also my collaborator H. Burkhardt for helping me in the preparation of this talk.

\section{REFERENCES}

1. LEP Electroweak Working Group, http://lepewwg.web.cern.ch/LEPEWWG/.

2. H. Burkhardt and B. Pietrzyk, Phys. Rev. D72:057501,2005, Phys. Lett. B513 (2001)46.

3. B. Pietrzyk, Proc. of the XXIX Rencontres de Moriond, EW Interactions, March 12-19, contributions at $m_{\mathrm{Z}} \quad$ Burkhardt, Pietrzyk 2005
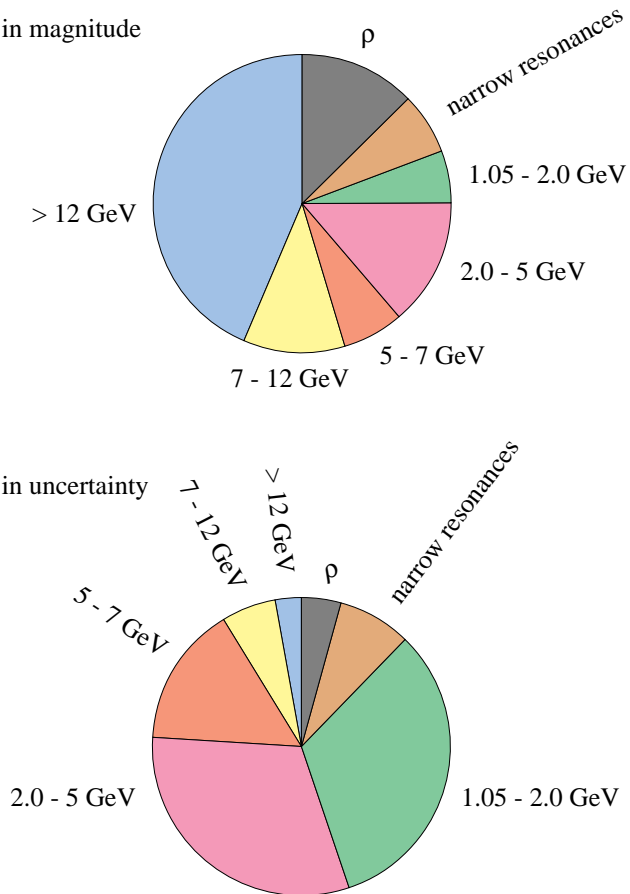

Figure 9. Relative contribution to $\Delta \alpha_{\text {had }}^{(5)}$ in magnitude and uncertainty.

1994, Ed. J. Trân Thanh Vân, page 137.

4. C. Edwards et al., Crystal Ball Collaboration, SLAC-PUB-5160, Jan. 1990.

5. J.L. Siegrist et al., MARKI Collaboration, Phys. Rev. D 26 (1982) 969.

6. Z.G. Zhao, Proc. of ICHEP 2000, Osaka, Japan, 27 July-2 August 2000, Ed. C.S. Lim and T. Yamanaka, page 644.

7. J.Z. Bai, BES Collaboration, Phys.Rev.Lett. 88:101802,2002.

8. B. Pietrzyk, Proc. of ICHEP 2000, Osaka, Japan, 27 July-2 August 2000, Ed. C.S. Lim and T. Yamanaka, page 710.

9. R.R. Akhmetshin et al., CMD-2 Collaboration, Phys. Lett. B578 (2004)285.

10. A. Aloisio et al., KLOE Collaboration, Phys. Lett. B606 (2005)12.

11. M.N. Achasov, SND Collaboration, J. Exp. Theor. Phys. 101:1053-1070, 2005.

12. M.N. Achasov, I. Logashenko, these proceedings. 